\title{
The mixed-type integral equations for transient elastodynamic plane crack problems
}

\author{
WEIJIANG CHEN ${ }^{1}$, CHUNTU LIU $^{1}$ and RENJI TANG ${ }^{2}$ \\ 'Institute of Mechanics, Chinese Academy of Sciences, Beijing 100080, China \\ ${ }^{2}$ Department of Engineering Mechanics, Shanghai Jiaotong University, Shanghai 200030, China
}

Received 28 November 1995; accepted in revised form 12 June 1996

\begin{abstract}
In the present paper, by use of the boundary integral equation method and the techniques of Green fundamental solution and singularity analysis, the dynamic infinite plane crack problem is investigated. For the first time, the problem is reduced to solving a system of mixed-typed integral equations in Laplace transform domain. The equations consist of ordinary boundary integral equations along the outer boundary and Cauchy singular integral equations along the crack line. The equations obtained are strictly proved to be equivalent with the dual integral equations obtained by Sih in the special case of dynamic Griffith crack problem. The mixed-type integral equations can be solved by combining the numerical method of singular integral equation with the ordinary boundary element method. Further use the numerical method for Laplace transform, several typical examples are calculated and their dynamic stress intensity factors are obtained. The results show that the method proposed is successful and can be used to solve more complicated problems.
\end{abstract}

\section{Introduction}

In fracture dynamics, the impact or transient loading problems possess high value of practice and theory. But due to mathematical difficulties, the solution of problems in classical elastodynamics remains an extremely complicated and difficult task. Only limited solutions in a closed form have been obtained for infinite domain problems. To obtain solutions for finite and irregular domains, there is a clear need for developing powerful theory and effective numerical techniques which can model arbitrary time-dependent loads and geometry.

In special transient crack problems, the Griffith crack problem may be the most basic one. This problem was firstly considered by Sih and Chen [1]. They applied the Laplace-Fourier integral transforms and obtained a system of dual integral equations. In 1980's the method was further applied to some other special problems [2-5]. In general ones, the pure numerical methods, finite difference method (FDM) and finite element method (FEM) have been applied with some success to solve dynamic problems of cracks. But some difficulties in using FDM and FEM for fracture dynamics have been pointed out [6]. The boundary integral equation method (BIEM) provides an efficient numerical approach towards crack analysis. However the conventional displacement BIE formulation gives rise to degenerate integral equations for crack problems and is not suitable for numerical solutions. For dynamic crack problem, the ordinary BIEM was firstly used by Fan and Hahn [7], Sladek and Sladek [8], Chirno and Dominguez [9], where the domain has to be divided into subdomains by means of a cut along the crack. To circumvent the difficulties in using the ordinary integral equation method, several other approaches have been proposed; see for example, the papers by Cruse [10], Sladek and Sladek [11], Nishimura and Kobayashi [12]. Most of these studies first reduced the high order singularities to integrable ones, and then solved the modified BIE's numerically. A simple and straightforward derivation of non-hypersingular BIEs for elastodynamic crack 
analysis was recently proposed by Zhang, Achenbach and Gross [13-16], where a two-state conservation integral of elastodynamics is used and the unknown quantities are the crack opening displacements and their derivatives. The dynamic crack problems of $2 \mathrm{D}$ and $3 \mathrm{D}$ infinite bodies have been solved by them in both the time domain and the frequency domain.

In the present paper, the finite plane crack problem subjected to impact loading is investigated. The traction BIEs are derived. Some techniques of the Green fundamental solution and singularity analysis are used to obtain Cauchy singular integral equations along the crack line with the crack dislocation densities as unknowns. Combining the ordinary BIE along the outer boundary, the problem is finally reduced to solving a set of mixed-type integral equations. By use of the ordinary BEM and the numerical methods for singular integral equations, the equations are firstly solved in Laplace transform domain, then the final solutions can be calculated by Laplace numerical inversion. In the last section of this paper, several typical examples are calculated and the dynamic stress intensity factors are obtained.

\section{The basic formulation}

In the linear elastodynamics problem, the displacement field $u_{i}(y, t)$ must satisfy the equations of motion

$$
\mu u_{i, k k}+(\lambda+\mu) u_{k, k i}=\rho \frac{\partial^{2} u_{i}}{\partial t^{2}}
$$

and boundary and initial conditions

$$
\begin{aligned}
& \left\{\begin{array}{l}
\sigma_{i j} n_{j}=t_{i}(x, t), x \in \Gamma_{t} \\
u_{i}=u_{i}^{0}(x, t), x \in \Gamma_{u}
\end{array}, \quad t \geqslant 0,\right. \\
& \left\{\begin{array}{l}
\left.u_{i}\right|_{t=0}=u_{i}^{0}(x), \\
\left.\frac{\partial u_{i}}{\partial t}\right|_{t=0}=\dot{u}_{i}^{0}(x)
\end{array}, \quad x \in \Omega+\Gamma,\right.
\end{aligned}
$$

where $\lambda$ and $\mu$ are elastic modules, $\rho$ is density of material and $\Omega$ is the region enclosed by boundary $\Gamma\left(\Gamma=\Gamma_{t}+\Gamma_{u}\right)$.

Supposing the initial conditions are homogeneous, that is $u_{i}^{0}=\dot{u}_{i}^{0}$, and applying the Laplace transform about time $(t)$ to $(1)$, we obtain

$$
\mu \vec{u}_{i, k k}+(\lambda+\mu) \bar{u}_{k, k i}-\rho p^{2} \bar{u}_{i}=0
$$

where the bar denotes the Laplace transform, $p$ is the transform parameter. The solution for (4) in $\Omega$ can be expressed by the Somigliana formula

$$
\bar{u}_{k}(y, p)=\int_{\Gamma}\left[\bar{t}_{i}(\eta, p) \bar{U}_{i k}(\eta-y, p)-\bar{u}_{i}(\eta, p) \bar{T}_{i k}(\eta, y, p)\right] \mathrm{d} \Gamma(\eta),
$$

where $\bar{U}_{i k}$ and $\bar{T}_{i k}$ are the Green fundamental solution (see Appendix).

\section{The mixed-type integral equations for the crack problem}

For the crack problem shown in Figure 1, the boundary consists of two parts $\Gamma=S+L^{ \pm}$, where $S$ is the outer boundary and $L^{ \pm}$is the surface of crack. Then (5) can be rewritten as 


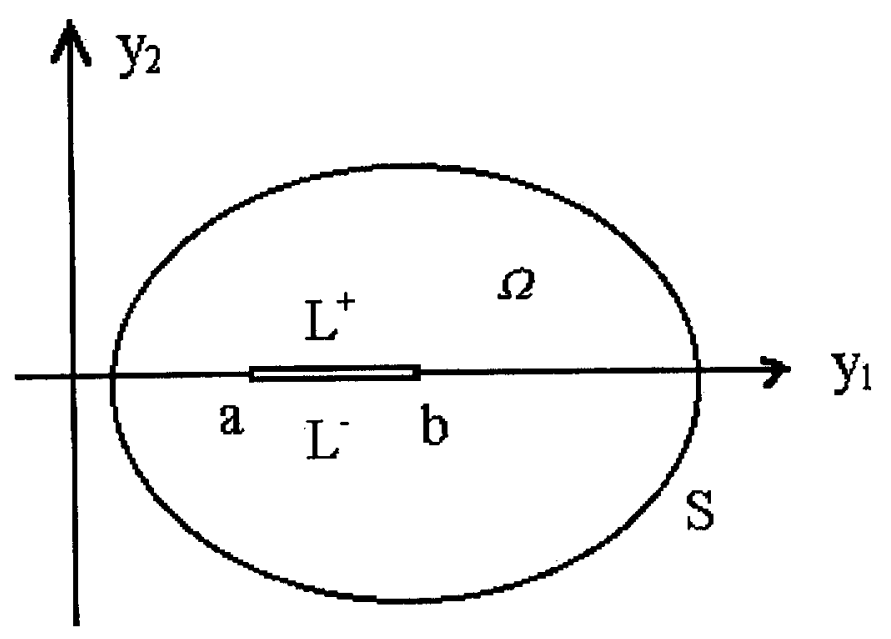

Figure 1. A plane crack problem.

$$
\begin{aligned}
\bar{u}_{k}(y, p)= & \int_{S}\left[\bar{t}_{i}(\eta, p) \vec{U}_{i k}(\eta-y, p)-\bar{u}_{i}(\eta, y, p) \bar{T}_{i k}(\eta, y, p)\right] \mathrm{d} \Gamma(\eta) \\
& -\int_{a}^{b} \Delta \bar{u}_{i}\left(\eta_{1}, p\right) \bar{T}_{i k}\left(\eta_{1}, y, p\right) \mathrm{d} \eta_{1}
\end{aligned}
$$

where $\Delta \bar{u}_{k}=\left.\bar{u}_{i}\right|_{L^{+}}-\left.\bar{u}_{i}\right|_{L^{-}}$is the dislocation between up and down of the crack. Let $n$ be the boundary outward normal, then

$$
\bar{T}_{i k}^{+}=\left.\bar{T}_{i k}\right|_{n(0,-1)}, \bar{T}_{i k}^{+}\left(\eta_{1}, y, p\right)=\left.\bar{T}_{i k}^{+}(\eta, y, p)\right|_{\eta_{2}=0}
$$

Let $y \rightarrow S$ in (6). One can obtain the regular boundary integral equations along the outer boundary $S$.

$$
\begin{aligned}
\frac{1}{2} \bar{u}_{k}(y, p)= & \int_{S}\left[\bar{t}_{i}(\eta, p) \bar{U}_{i k}(\eta-y, p)-\bar{u}_{i}(\eta, y, p) \bar{T}_{i k}(\eta, y, p)\right] \mathrm{d} \Gamma(\eta) \\
& -\int_{a}^{b}\left[\int_{\eta_{1}}^{b} \bar{T}_{i k}\left(\eta^{*}, y, p\right) \mathrm{d} \eta^{*}\right] \Delta \bar{u}_{i, 1}\left(\eta_{1}, p\right) \mathrm{d} \eta_{1}, \quad y \in S
\end{aligned}
$$

where the close condition at crack-tip $\left.\Delta \bar{u}_{i, 1}\right|_{a^{+}, b^{-}}=0$ has been used and $\Delta \bar{u}_{i, 1}=\partial \Delta \bar{u}_{i} / \partial \eta_{1}$ is the dislocation density function along the crack.

In order to obtain the traction BIEs along the crack, some techniques have to be used. By the meaning of Green's fundamental solution, the following equations are satisfied

$$
\frac{\partial \bar{T}_{i k}^{+}(\eta, y, p)}{\partial y_{2}}=-\frac{\partial \bar{W}_{i k}(\eta, y, p)}{\partial \eta_{1}}+\rho p^{2} \bar{U}_{i k}(\eta, y, p), \quad \eta \neq y
$$

where $\bar{W}_{i k}(\eta, y, p)=\left.\bar{T}_{i k}(\eta, y, p)\right|_{n(1,0)}$.

Substitute (6) into physical equations

$$
\bar{\sigma}_{k 2}=\mu\left(\bar{u}_{1,2}+\bar{u}_{2,1}\right), \quad \bar{\sigma}_{22}=\frac{2 \mu}{1-2 \nu}\left[\nu \bar{u}_{1,1}+(1-\nu) \bar{u}_{2,2}\right]
$$


Weijiang Chen et al.

and use (8) and integrate by part, the stresses in $\Omega$ can be expressed as

$$
\begin{aligned}
\bar{\sigma}_{k 2}(y, p)= & \int_{S}\left[\bar{t}_{i}(\eta, p) \bar{T}_{k i}^{+}(\eta, y, p)+\bar{K}_{i k}(\eta, y, p) \bar{u}_{i}(\eta, p)\right] \mathrm{d} \Gamma(\eta) \\
& +\int_{a}^{b} H_{i k}(\eta, y, p) \Delta \bar{u}_{i, 1}\left(\eta_{1}, p\right) \mathrm{d} \eta_{1}
\end{aligned}
$$

in which

$$
\begin{aligned}
& \bar{K}_{i 1}=-\mu\left(\frac{\partial \bar{T}_{i 1}}{\partial y_{2}}+\frac{\partial \bar{T}_{i 2}}{\partial y_{1}}\right) \\
& \bar{K}_{i 2}=-\frac{2 \mu}{1-2 \nu}\left[\nu \frac{\partial \bar{T}_{i 1}}{\partial y_{1}}+(1-\nu) \frac{\partial \bar{T}_{i 2}}{\partial y_{2}}\right] \\
& H_{i 1}=-\mu\left(\bar{T}_{i 2}+\bar{W}_{i 1}\right)-\mu \rho p^{2} \int_{\eta_{1}}^{b} \bar{U}_{i 1} \mathrm{~d} \eta^{*} \\
& H_{i 2}=-\frac{2 \mu}{1-2 \nu}\left[\nu \bar{T}_{i 1}^{+}+(1-\nu) \bar{W}_{i 2}\right]-\frac{2 \mu(1-\nu)}{1-2 \nu} \rho p^{2} \int_{\eta_{1}}^{b} \bar{U}_{i 2} \mathrm{~d} \eta^{*} .
\end{aligned}
$$

From [8], the Green fundamental solutions of dynamics and statics satisfy the following asymptotic relations

$$
\bar{T}_{i k}-T_{i k}=O(|\eta-y| \ln |\eta-y|), \quad \bar{U}_{i k}-U_{i k}=O(1),
$$

where $U_{i k}$ and $T_{i k}$ are static Green fundamental solutions (also see Appendix). It shows that the dynamic and static problems have the same singularity. Let $y \rightarrow L^{ \pm}$in (10) and use relations (11) to separate the singular static part from the integral kernels. We finally obtain the Cauchy singular integral equations along the crack line $(a, b)$

$$
\begin{aligned}
\int_{S} & {\left[\bar{t}_{i}(\eta, p) \bar{T}_{k i}^{+}\left(\eta, y_{1}, p\right)+\bar{K}_{i k}(\eta, y, p) \bar{u}_{i}(\eta, p)\right] \mathrm{d} S(\eta) } \\
& +\frac{A}{\pi} \int_{a}^{b} \frac{\Delta \bar{u}_{k, 1}\left(\eta_{1}, p\right)}{\eta_{1}-y_{1}} \mathrm{~d} \eta_{1}+\int_{a}^{b} \bar{H}_{i k}\left(\eta_{1}, y_{1}, p\right) \Delta \bar{u}_{i, 1}\left(\eta_{1}, p\right) \mathrm{d} \eta_{1} \\
= & \bar{q}_{k}\left(y_{1}, p\right), \quad k=1,2, \quad y_{1} \in(a, b),
\end{aligned}
$$

in which, $\bar{q}_{k}\left(y_{1}, p\right)=\left.\bar{\sigma}_{k 2}\right|_{L^{ \pm}}$is the Laplace transform of known dynamic loading on crack surface, and

$$
\begin{gathered}
A=\mu / 2(1-\nu), \quad \bar{H}_{12}\left(\eta_{1}, y_{1}, p\right)=\bar{H}_{21}\left(\eta_{1}, y_{1}, p\right)=0 \\
\bar{H}_{11}=-\mu\left(\bar{T}_{12}^{+}+\bar{W}_{11}-T_{12}-W_{11}\right)-\mu \rho p^{2} \int_{\eta_{1}}^{b} \bar{U}_{11} \mathrm{~d} \eta^{*} \\
\bar{H}_{22}=-\frac{2 \mu}{1-2 \nu}\left[\nu\left(\bar{T}_{21}^{+}-T_{21}\right)+(1-\nu)\left(\bar{W}_{22}-W_{22}\right)\right] \\
-\frac{2 \mu(1-\nu)}{1-2 \nu} \rho p^{2} \int_{\eta_{1}}^{b} \bar{U}_{22} \mathrm{~d} \eta^{*}
\end{gathered}
$$


where $\nu$ is the Poisson ratio and should be substituted by $\nu /(1-\nu)$ in plane stress problem. Now the problem is reduced to mixed-type integral Equations (7) and (12). To solve them, the following single value conditions of displacement are needed

$$
\int_{a}^{b} \Delta \bar{u}_{i, 1}\left(\eta_{1}, p\right) \mathrm{d} \eta_{1}=0, \quad i=1,2
$$

The solutions for the above equations belong to the Laplace transform domain. After the Laplace transform inversion one can obtain a solution of the problem. For the special Griffith crack problem, we can simply obtain the Cauchy singular integral equations by letting $S \rightarrow \infty$

$$
\begin{aligned}
& \frac{A}{\pi} \int_{a}^{b} \frac{\Delta \bar{u}_{1,1}}{\eta_{1},-y_{1}} \mathrm{~d} \eta_{1}+\int_{a}^{b} \bar{H}_{11} \Delta \bar{u}_{1,1} \mathrm{~d} \eta_{1}=\bar{q}_{1}\left(y_{1}, p\right), \\
& \frac{A}{\pi} \int_{a}^{b} \frac{\Delta \bar{u}_{2,1}}{\eta_{1}-y_{1}} \mathrm{~d}_{1}+\int_{a}^{b} \bar{H}_{22} \Delta \bar{u}_{2,1} \mathrm{~d} \eta_{1}=\bar{q}_{2}\left(y_{1}, p\right),
\end{aligned}
$$

where (14b) is for type-I problem and (14a) is for type-II problem. They are clearly independent and can be easily used to for multi-crack problems.

The above method can be used for static problems in the similar way, where we only need to exchange the dynamic Green fundamental solution for a static one and remove the inertial terms. The integral equations for static problems are listed as follows

$$
\begin{aligned}
& \frac{1}{2} u_{k}(y)= \int_{S}\left[t_{i}(\eta) U_{i k}(\eta, y)-u_{i}(\eta) T_{i k}(\eta, y)\right] \mathrm{d} S(\eta) \\
& \quad-\int_{a}^{b}\left[\int_{\eta_{1}}^{b} T_{i k}^{+}\left(\eta^{*}, y\right) \mathrm{d} \eta^{*}\right] \Delta u_{i, 1}\left(\eta_{1}\right) \mathrm{d} \eta_{1}, \quad y \in S, \\
& \int_{S}\left[t_{i}(\eta) T_{k i}^{+}\left(\eta, y_{1}\right)+K_{i 1}\left(\eta, y_{1}\right) u_{i}(\eta)\right] \mathrm{d} S(\eta) \\
&+\frac{A}{\pi} \int_{a}^{b} \frac{\Delta u_{k, 1}}{\eta_{1}-y_{1}} \mathrm{~d} \eta_{1}=q_{k}\left(y_{1}\right), \quad y_{1} \in(a, b), \quad k=1,2,
\end{aligned}
$$

where

$$
\begin{aligned}
& T_{i k}^{+}=\left.T_{i k}\right|_{n(0,-1)}, \quad K_{i 1}=-\mu\left(\frac{\partial T_{i 1}}{\partial y_{2}}+\frac{\partial T_{i 2}}{\partial y_{1}}\right), \\
& K_{i 2}=-\frac{2 \mu}{1-2 \nu}\left[\nu \frac{\partial T_{i 1}}{\partial y_{1}}+(1-\nu) \frac{\partial T_{i 2}}{\partial y_{2}}\right] .
\end{aligned}
$$

\section{The equivalence proof between two kinds of equations}

In this section we will prove that Equation (14b) proposed in the present paper is equivalent to the equations obtained by Sih and Chen [1], where, by the use of Laplace-Fourier transforms, the Griffith type-I crack $\left(-a_{0}, a_{1}\right)$ problem is reduced to solve the dual integral equations as

$$
\int_{0}^{\infty} C(\beta, p) \cos \left(\beta y_{1}\right) \mathrm{d} \beta=0, \quad y_{1}>a_{0},
$$


Weijiang Chen et al.

$$
\int_{0}^{\infty} f(\beta, p) C(\beta, p) \cos \left(\beta y_{1}\right) \mathrm{d} \beta=\bar{q}_{2}\left(y_{1}, p\right), \quad 0<y_{1}<a_{0}
$$

where

$$
\begin{aligned}
& f(\beta, p)=-\frac{1}{\pi} \frac{\mu}{\varsigma_{1}}\left\{\left[2 \beta^{2}+\left(\frac{p}{c_{2}}\right)^{2}\right]^{2}-4 \beta^{2} \varsigma_{1} \varsigma_{2}\right\}, \\
& \varsigma_{1}=\sqrt{\beta^{2}+\left(\frac{p}{c_{1}}\right)^{2}}, \quad c_{1}=\sqrt{\frac{\lambda+2 \mu}{\rho}} \\
& \varsigma_{2}=\sqrt{\beta^{2}+\left(\frac{p}{c_{2}}\right)^{2}}, \quad c_{2}=\sqrt{\frac{\mu}{\rho}}
\end{aligned}
$$

To prove the equivalence, at first we need to simplify the dual integral Equations $(16 \mathrm{a}, \mathrm{b})$. Since it has been supposed that the problem is symmetric about $y_{2}$ axis [1], the loading $q_{2}\left(y_{1}, t\right)$ satisfies condition $q_{2}\left(y_{1}, t\right)=q_{2}\left(-y_{1}, t\right)$. The displacement can be expressed by the unknown function $C(\beta, p)$

$$
\begin{aligned}
\bar{u}_{2}\left(y_{1}, p\right)= & \frac{2}{\pi} \int_{0}^{\infty}\left\{\frac{1}{2}\left[2 \beta^{2}+\left(\frac{p}{c_{2}}\right)^{2}\right] \mathrm{e}^{-\varsigma_{1} y_{2}}-\beta^{2} \mathrm{e}^{-\varsigma_{2} y_{2}}\right\} \\
& \times \cos \left(\beta y_{1}\right) C(\beta, p) \mathrm{d} \beta .
\end{aligned}
$$

Then the dislocation density is

$$
\frac{1}{2} \Delta \bar{u}_{2,1}=\left.\frac{\partial \bar{u}_{2}}{\partial y_{1}}\right|_{y_{2}=0^{+}}=-\frac{1}{\pi} \int_{0}^{\infty} \beta\left(\frac{p}{c_{2}}\right)^{2} \sin \left(\beta y_{1}\right) C(\beta, p) \mathrm{d} \beta .
$$

Appling the Fourier sine integral transform to above equation, we obtain

$$
C(\beta, p)=-\frac{1}{\beta}\left(\frac{c_{2}}{p}\right)^{2} \int_{0}^{a_{0}} \sin \left(\beta \eta_{1}\right) \Delta \bar{u}_{2,1}\left(\eta_{1}, p\right) \mathrm{d} \eta_{1},
$$

Substitute (20) into the left of (16a)

$$
\begin{aligned}
\int_{0}^{\infty} & C(\beta, p) \cos \left(\beta y_{1}\right) \mathrm{d} \beta \\
= & -\frac{1}{2}\left(\frac{c_{2}}{p}\right)^{2} \int_{0}^{a_{0}}\left\{\int_{0}^{\infty}\left[\frac{\sin \beta\left(\eta_{1}+y_{1}\right)}{\beta}-\frac{\sin \beta\left(\eta_{1}-y_{1}\right)}{\beta}\right] \mathrm{d} \beta\right\} \\
& \times \Delta \bar{u}_{2,1}\left(\eta_{1}, p\right) \mathrm{d} \eta_{1},
\end{aligned}
$$

and use formula

$$
\int_{0}^{\infty} \frac{\sin \alpha \beta}{\beta} \mathrm{d} \beta=\frac{1}{2} \pi, \quad \alpha>0
$$


Paying attention to condition $y_{1}>a_{0}>\eta_{1}>0$ in (21), one can easily examine that (16a) is satisfied automatically. Also, substitute (20) and (17) into (16b)

$$
\frac{1}{\pi} \int_{0}^{a_{1}} L\left(\eta_{1}, y_{1}, p\right) \Delta \bar{u}_{2,1}\left(\eta_{1}, p\right) \mathrm{d} \eta_{1}=\bar{q}_{2}\left(y_{1}, p\right), \quad 0<y_{1}<a_{0},
$$

where the kernel is

$$
\begin{aligned}
L\left(\eta_{1}, y_{1}, p\right)= & \frac{\mu}{\rho p^{2}} \int_{0}^{\infty}\left[2 \mu \frac{\beta^{3}}{\varsigma_{1}}+2 \rho p^{2} \frac{1}{\beta \varsigma_{1}}+\frac{\left(\rho p^{2}\right)^{2}}{2 \mu \beta \varsigma_{1}}-2 \mu \beta \varsigma_{2}\right] \\
& \times\left[\sin \beta\left(\eta_{1}-y_{1}\right)+\sin \beta\left(\eta_{1}+y_{1}\right)\right] \mathrm{d} \beta .
\end{aligned}
$$

To simplify this kernel, we use the following integral variable substitutions respectively

$$
\beta=\frac{p}{c_{1}} \text { sh } \varsigma, \quad \beta=\frac{p}{c_{2}} \text { sh } \varsigma .
$$

Then kernel (24) can be changed into

$$
\begin{aligned}
L\left(\eta_{1}, y_{1}, p\right) & \\
= & \frac{\mu}{\rho p^{2}} \int_{0}^{\infty}\left[2 \mu\left(\frac{p}{c_{1}}\right)^{3} s h^{3} \varsigma+2 \rho p^{2} \frac{p}{c_{1}} s h \varsigma\right] \\
& \times\left[\sin \frac{p\left(\eta_{1}-y_{1}\right) s h \varsigma}{c_{1}}+\sin \frac{p\left(\eta_{1}+y_{1}\right) s h \varsigma}{c_{1}}\right] \mathrm{d} \varsigma \\
& -\frac{\mu}{\rho p^{2}} \int_{0}^{\infty} 2 \mu\left(\frac{p}{c_{2}}\right)^{2}\left[\sin \frac{p\left(\eta_{1}-y_{1}\right) s h \varsigma}{c_{1}}+\sin \frac{p\left(\eta_{1}+y_{1}\right) s h \varsigma}{c_{1}}\right] \operatorname{ch} \varsigma \mathrm{d} \varsigma \\
& +\frac{1}{2} \rho p^{2} \int_{0}^{\infty} \frac{\sin \beta\left(\eta_{1}-y_{1}\right)}{\beta \varsigma_{1}} \mathrm{~d} \beta+\frac{1}{2} \rho p^{2} \int_{0}^{\infty} \frac{\sin \beta\left(\eta_{1}+y_{1}\right)}{\beta \varsigma_{1}} \mathrm{~d} \beta,
\end{aligned}
$$

by use of the integral expression and differential relation for Bessel function and their derivative

$$
\begin{aligned}
& K_{0}(Z)=\int_{0}^{\infty} \cos (Z s h \varsigma) \mathrm{d} \varsigma, \quad K_{1}(Z)=\int_{0}^{\infty} \sin (Z s h \varsigma) \operatorname{sh} \varsigma \mathrm{d} \varsigma, \\
& \frac{\mathrm{d} K_{1}(Z)}{\mathrm{d} Z}=-K_{0}(Z)-\frac{1}{Z} K_{1}(Z), \quad \operatorname{Re}(Z)>0 .
\end{aligned}
$$

The kernel can be rewritten as

$$
\begin{aligned}
& L\left(\eta_{1}, y_{1}, p\right)=r_{, 1}\{-\frac{2 \mu^{2}}{\rho p^{2}}\left(\frac{p}{c_{1}}\right)^{3} K_{1}^{\prime \prime}\left(\frac{p r}{c_{1}}\right)+\frac{2 \mu p}{c_{1}} K_{1}\left(\frac{p r}{c_{1}}\right) \\
&\left.+\frac{4 \mu^{2}}{\rho p^{2}}\left(\frac{p}{c_{2}}\right)^{3}\left[K_{1}^{\prime \prime}\left(\frac{p r}{c_{2}}\right)-K_{1}\left(\frac{p r}{c_{2}}\right)\right]\right\} \\
&+\bar{r}_{, 1}\left\{-\frac{2 \mu^{2}}{\rho p^{2}}\left(\frac{p}{c_{1}}\right)^{3} K_{1}^{\prime \prime}\left(\frac{p \bar{r}}{c_{1}}\right)+\frac{2 \mu p}{c_{1}} K_{1}\left(\frac{p \bar{r}}{c_{1}}\right)\right.
\end{aligned}
$$


Weijiang Chen et al.

$$
\begin{gathered}
\left.+\frac{4 \mu^{2}}{\rho p^{2}}\left(\frac{p}{c_{2}}\right)^{3}\left[K_{1}^{\prime \prime}\left(\frac{p \bar{r}}{c_{2}}\right)-K_{1}\left(\frac{p \bar{r}}{c_{2}}\right)\right]\right\} \\
+\frac{1}{2} \rho p^{2} \int_{0}^{\infty} \frac{\sin \beta\left(\eta_{1}-y_{1}\right)}{\beta \varsigma_{1}} \mathrm{~d} \beta+\frac{1}{2} \rho p^{2} \int_{0}^{\infty} \frac{\sin \beta\left(\eta_{1}+y_{1}\right)}{\beta \varsigma_{1}} \mathrm{~d} \beta,
\end{gathered}
$$

where $r=\left|\eta_{1}-y_{1}\right|, \bar{r}=\left|\eta_{1}+y_{1}\right|, r_{, 1}=\left(\eta_{1}-y_{1}\right) / r, \bar{r}_{, 1}=\left(\eta_{1}-y_{1}\right) / \bar{r}$ and by further use of relation $27(\mathrm{~b})$, the kernel is finally rewritten as

$$
\begin{aligned}
L\left(\eta_{1}, y_{1}, p\right)= & -\frac{2 \mu r_{, 1}}{r}\left(\frac{c_{2}}{c_{1}}\right)^{2} K_{0}\left(\frac{p r}{c_{1}}\right)-\frac{4 \mu r_{, 1}}{p r^{2}} \frac{c_{2}^{2}}{c_{1}} K_{1}\left(\frac{p r}{c_{1}}\right) \\
& +\frac{2 \mu p r_{, 1}}{c_{1}}\left(-\frac{c_{2}^{2}}{c_{1}^{2}}+1\right) K_{1}\left(\frac{p r}{c_{1}}\right)+\frac{2 \mu r_{, 1}}{r} K_{0}\left(\frac{p r}{c_{2}}\right) \\
& +\frac{4 \mu r_{, 1}}{p r^{2}} c_{2} K_{1}\left(\frac{p r}{c_{2}}\right)-\frac{2 \mu \bar{r}, 1}{\bar{r}}\left(\frac{c_{2}}{c_{1}}\right)^{2} K_{0}\left(\frac{p \bar{r}}{c_{1}}\right) \\
& -\frac{4 \mu \bar{r}_{, 1}}{p \bar{r}^{2}} \frac{c_{2}^{2}}{c_{1}} K_{1}\left(\frac{p \bar{r}}{c_{1}}\right)+\frac{2 \mu p \bar{r}_{, 1}}{c_{1}}\left(-\frac{c_{2}^{2}}{c_{1}^{2}}+1\right) K_{1}\left(\frac{p \bar{r}}{c_{1}}\right) \\
& +\frac{2 \mu \bar{r}_{, 1}}{\bar{r}} K_{0}\left(\frac{p \bar{r}}{c_{2}}\right) \frac{4 \mu \bar{r}_{, 1}}{p \bar{r}^{2}} c_{2} K_{1}\left(\frac{p \bar{r}}{c_{2}}\right) \\
& +\frac{1}{2} \rho p^{2} \int_{0}^{\infty} \frac{\sin \beta\left(\eta_{1}-y_{1}\right)}{\beta \varsigma_{1}} \mathrm{~d} \beta+\frac{1}{2} \rho p^{2} \int_{0}^{\infty} \frac{\sin \beta\left(\eta_{1}+y_{1}\right)}{\beta \varsigma_{1}} \mathrm{~d} \beta
\end{aligned}
$$

Now the dual integral Equations (16a,b) have been changed into (23) with kernel (29), then we need to prove that (14) is equivalent with (23). Paying attention to the symmetry, (14) can be changed into

$$
\frac{1}{\pi} \int_{0}^{a_{1}} G\left(\eta_{1}, y_{1}, p\right) \Delta \bar{u}_{2,1}\left(\eta_{1}, p\right) \mathrm{d} \eta_{1}=\bar{q}_{2}\left(y_{1}, p\right)
$$

where the kernel is

$$
\begin{aligned}
G\left(\eta_{1}, y_{1}, p\right)= & -\frac{2 \mu \pi}{1-2 \nu}\left[\mu \bar{T}_{21}^{+}\left(\eta_{1}, y_{1}, p\right)+(1-\nu) \bar{W}_{22}\left(\eta_{1}, y_{1}, p\right)\right] \\
& -\frac{2 \mu \pi}{1-2 \nu}\left[\mu \bar{T}_{21}^{+}\left(\eta_{1},-y_{1}, p\right)+(1-\nu) \bar{W}_{22}\left(\eta_{1},-y_{1}, p\right)\right] \\
& -\frac{2 \mu(1-\nu) \pi \rho p^{2}}{1-2 \nu} \int_{\eta_{1}}^{a_{0}} \bar{U}_{22}\left(\eta^{*}-y_{1}, p\right) \mathrm{d} \eta^{*} \\
& -\frac{2 \mu(1-\nu) \pi \rho p^{2}}{1-2 \nu} \int_{\eta_{1}}^{-a_{0}} \bar{U}_{22}\left(\eta^{*}+y_{1}, p\right) \mathrm{d} \eta^{*}
\end{aligned}
$$


Now we only need to prove that (23) and (30) have the same kernel, that is $L\left(\eta_{1}, y_{1}, p\right)=$ $G\left(\eta_{1}, y_{1}, p\right)$. Substituting the expressions of Green's fundamental solution to (31), after careful derivation we can obtain

$$
\begin{aligned}
& G\left(\eta_{1}, y_{1}, p\right)=-\frac{2 \mu r_{, 1}}{r}\left(\frac{c_{2}}{c_{1}}\right)^{2} K_{0}\left(\frac{p r}{c_{1}}\right)-\frac{4 \mu r_{1}}{p r^{2}} \frac{c_{2}^{2}}{c_{1}} K_{1}\left(\frac{p r}{c_{1}}\right) \\
& -\frac{\nu \mu r_{, 1}}{1-2 \nu}\left(1-2 \frac{c_{2}^{2}}{c_{1}^{2}}\right) \frac{p}{c_{1}} K_{1}\left(\frac{p r}{c_{1}}\right) \\
& +\frac{2 \mu r_{, 1}}{r} K_{0}\left(\frac{p r}{c_{2}}\right)+\frac{4 \mu r_{, 1}}{p r^{2}} c_{2} K_{1}\left(\frac{p r}{c_{2}}\right)-\frac{(1-\nu) \mu r_{, 1}}{1-2 \nu} \frac{p}{c_{2}} K_{1}\left(\frac{p r}{c_{2}}\right) \\
& -\frac{1-\nu}{1-2 \nu} \rho p^{2} \int_{\eta_{1}}^{a_{0}}\left[K_{0}\left(\frac{p r^{*}}{c_{2}}\right)+\frac{c_{2}}{p r^{*}} K_{1}\left(\frac{p r^{*}}{c_{2}}\right)\right. \\
& \left.-\left(\frac{c_{2}}{c_{1}}\right)^{2} \frac{c_{1}}{p r^{*}} K_{1}\left(\frac{p r^{*}}{c_{1}}\right)\right] \mathrm{d} \eta^{*} \\
& -\frac{2 \mu \bar{r}_{, 1}}{\bar{r}}\left(\frac{c_{2}}{c_{1}}\right)^{2} K_{0}\left(\frac{p \bar{r}}{c_{1}}\right)-\frac{4 \mu \bar{r}, 1}{p \bar{r}^{2}} \frac{c_{2}^{2}}{c_{1}} K_{1}\left(\frac{p \bar{r}}{c_{1}}\right) \\
& -\frac{\nu \mu \bar{r}, 1}{1-2 \nu}\left(1-2 \frac{c_{2}^{2}}{c_{1}^{2}}\right) \frac{p}{c_{1}} K_{1}\left(\frac{p \bar{r}}{c_{1}}\right) \\
& +\frac{2 \mu \bar{r}_{, 1}}{\bar{r}} K_{0}\left(\frac{p \bar{r}}{c_{2}}\right)+\frac{4 \mu \bar{r}_{, 1}}{p \bar{r}^{2}} c_{2} K_{1}\left(\frac{p \bar{r}}{c_{2}}\right)-\frac{(1-\nu) \mu \bar{r}_{, 1}}{1-2 \nu} \frac{p}{c_{2}} K_{1}\left(\frac{p \bar{r}}{c_{2}}\right) \\
& -\frac{1-\nu}{1-2 \nu} \rho p^{2} \int_{\eta_{1}}^{-a_{o}}\left[K_{0}\left(\frac{p \bar{r}^{*}}{c_{2}}\right)+\frac{c_{2}}{p \bar{r}^{*}} K_{1}\left(\frac{p \bar{r}^{*}}{c_{2}}\right)\right. \\
& \left.-\left(\frac{c_{2}}{c_{1}}\right)^{2} \frac{c_{1}}{p \bar{r}^{*}} K_{1}\left(\frac{p \bar{r}^{*}}{c_{1}}\right)\right] \mathrm{d} \eta^{*}
\end{aligned}
$$

where $r^{*}=\left|\eta^{*}-y_{1}\right|, \bar{r}^{*}=\left|\eta^{*}+y_{1}\right|$. In order to simplify (32), by use of the relation (27b), we can easily examine that

$$
\begin{aligned}
& \frac{\mu p r_{, 1}}{c_{2}} K_{2}\left(\frac{p r}{c_{2}}\right)+\frac{\mu p \bar{r}_{, 1}}{c_{2}} K_{2}\left(\frac{p \bar{r}}{c_{2}}\right) \\
& =\rho p^{2} \int_{\eta_{1}}^{a_{0}}\left[K_{0}\left(\frac{p r^{*}}{c_{2}}\right)+\frac{c_{2}}{p r^{*}} K_{1}\left(\frac{p r^{*}}{c_{2}}\right)\right] \mathrm{d} \eta^{*} \\
& \quad+\rho p^{2} \int_{\eta_{1}}^{-a_{0}}\left[K_{0}\left(\frac{p \bar{r}^{*}}{c_{2}}\right)+\frac{c_{2}}{p \bar{r}^{*}} K_{1}\left(\frac{p \bar{r}^{*}}{c_{2}}\right)\right] \mathrm{d} \eta^{*}
\end{aligned}
$$

Then using $(26,32,33)$ and deriving carefully, we can obtain $L\left(\eta_{1}, y_{1}, P\right)-G\left(\eta_{1}, y_{1}, P\right)$ 


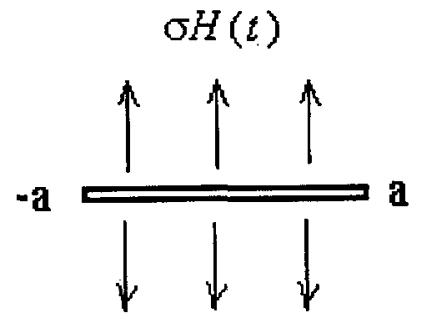

(a)

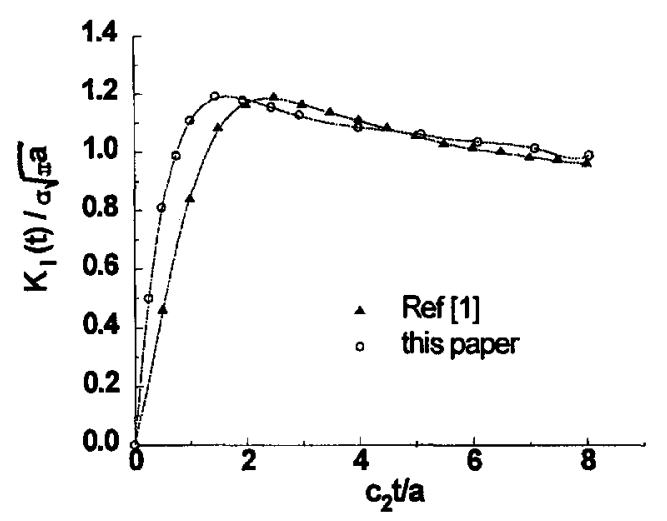

(b)

Figure 2. (a) Type-I Griffith crack problem. (b) The dynamic stress intensity factors.

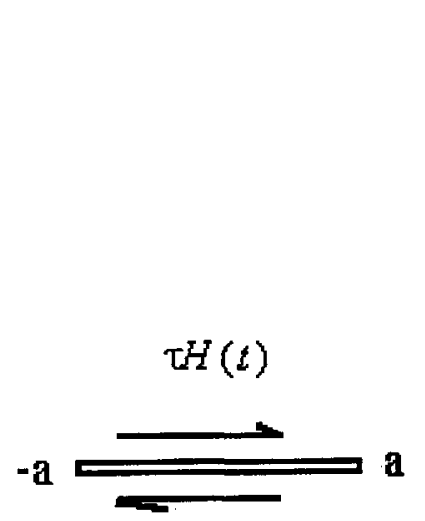

(a)

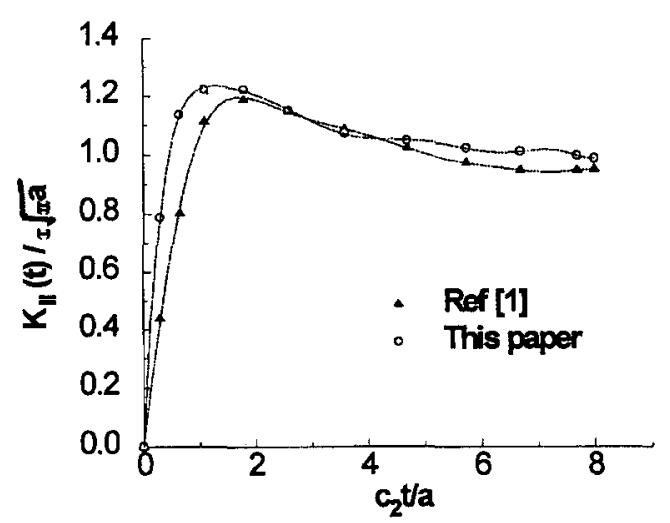

(b)

Figure 3. (a) Type-II Griffith crack problem. (b) The dynamic stress intensity factors.

$$
\begin{aligned}
= & \frac{1}{2} \rho p^{2} \int_{0}^{\infty}\left[\frac{\sin \beta\left(\eta_{1}-y_{1}\right)}{\beta \varsigma_{1}}+\frac{\sin \beta\left(\eta_{1}+y_{1}\right)}{\beta \varsigma_{1}}\right] \mathrm{d} \beta \\
& +\frac{\rho c_{1} p r_{, 1}}{2} K_{1}\left(\frac{p r}{c_{1}}\right)-\frac{1}{2} \rho p^{2} \int_{\eta_{1}}^{a_{0}} \frac{c_{1}}{p r^{*}} K_{1}\left(\frac{p r^{*}}{c_{1}}\right) \mathrm{d} \eta^{*} \\
& +\frac{\rho c_{1} p \bar{r}_{, 1}}{2} K_{1}\left(\frac{p \bar{r}}{c_{1}}\right)-\frac{1}{2} \rho p^{2} \int_{\eta_{1}}^{a_{0}} \frac{c_{1}}{p \bar{r}^{*}} K_{1}\left(\frac{p \bar{r}^{*}}{c_{1}}\right) \mathrm{d} \eta^{*}
\end{aligned}
$$

Also use relation

$$
K_{0}\left(\frac{p r}{c_{1}}\right)=\int_{0}^{\infty} \cos \left(\frac{p r}{c_{1}} s h \varsigma\right) \mathrm{d} \varsigma .
$$

We can easily examine that 


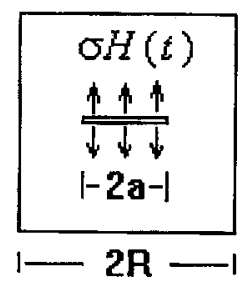

(a)

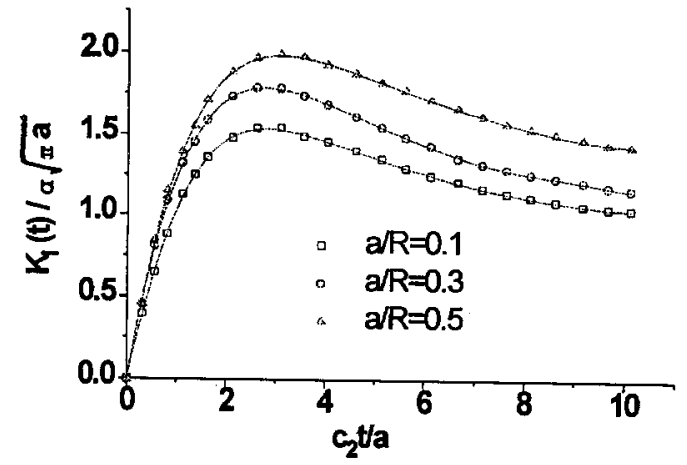

(b)

Figure 4. (a) Square with a central crack subjected to impact loading. (b) The dynamic stress intensity factors.

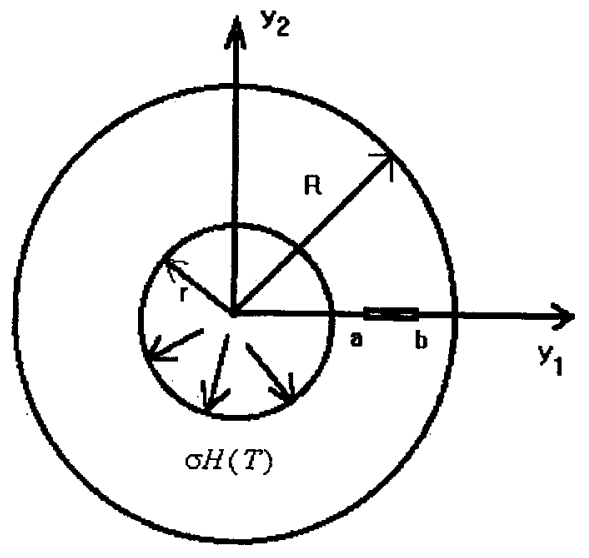

(a)

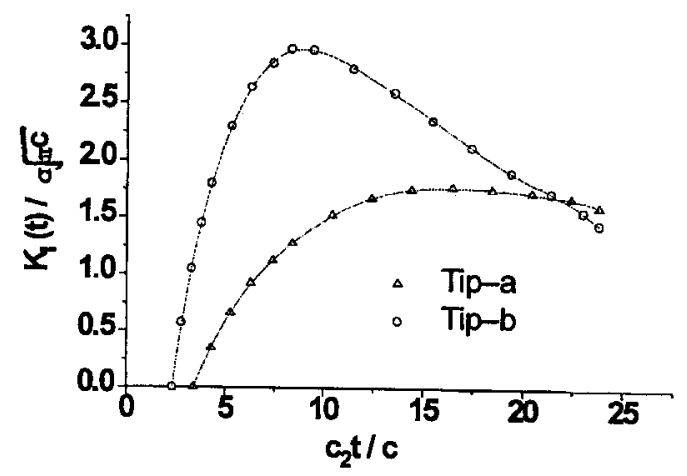

(b)

Figure 5. (a) Circular ring with a crack subjected to impact internal pressure. (b) The dynamic stress intensity factors.

$$
\begin{aligned}
& \int_{0}^{\infty}\left[\frac{\sin \beta\left(\eta_{1}-y_{1}\right)}{\beta \varsigma_{1}}+\frac{\sin \beta\left(\eta_{1}+y_{1}\right)}{\beta \varsigma_{1}}\right] \mathrm{d} \beta \\
& =\int_{\eta_{1}}^{a_{1}} \frac{c_{1}}{p r^{*}} K_{1}\left(\frac{p r^{*}}{c_{1}}\right) \mathrm{d} \eta^{*}+\int_{\eta_{1}}^{-a_{0}} \frac{c_{1}}{p \bar{r}^{*}} K_{1}\left(\frac{p \bar{r}^{*}}{c_{1}}\right) \mathrm{d} \eta^{*} .
\end{aligned}
$$

Then (34) can be reduced to

$$
\begin{gathered}
L\left(\eta_{1}, y_{1}, P\right)-G\left(\eta_{1}, y_{1}, P\right) \\
\quad=\frac{1}{2} \rho p^{2} \frac{c_{1} r, 1}{p} K_{1}\left(\frac{p r}{c_{1}}\right)
\end{gathered}
$$


Weijiang Chen et al.

$$
\begin{aligned}
& -\frac{1}{2} \rho p^{2} \int_{\eta_{1}}^{a_{0}}\left[K_{0}\left(\frac{p r^{*}}{c_{2}}\right)+\frac{c_{2}}{p r^{*}} K_{1}\left(\frac{p r^{*}}{c_{2}}\right)\right] \mathrm{d} \eta^{*} \\
= & \frac{1}{2} \rho p^{2} \frac{c_{1} \bar{r}_{, 1}}{p} K_{1}\left(\frac{p \bar{r}}{c_{1}}\right) \\
& -\frac{1}{2} \rho p^{2} \int_{\eta_{1}}^{-a_{0}}\left[K_{0}\left(\frac{p \bar{r}^{*}}{c_{2}}\right)+\frac{c_{2}}{p \bar{r}^{*}} K_{1}\left(\frac{p \bar{r}^{*}}{c_{2}}\right)\right] \mathrm{d} \eta^{*} .
\end{aligned}
$$

Use relation (27b) again, we obtain

$$
L\left(\eta_{1}, y_{1}, p\right)=G\left(\eta_{1}, y_{1}, p\right)
$$

It indicates that $(14)$ is equivalent with $(16 a, b)$ and shows that the method proposed in the present paper is reliable.

\section{The numerical methods}

Usually the analytical solutions to the integral equations of this paper are not available and it is therefore necessary to solve the equations numerically. By combining the numerical method of singular integral equation [17] with the boundary element method, a numerical method for the mixed-type integral equations is suggested, where the integrals along the outer boundary $S$ are calculated by BEM and the integrals along the crack line $(a, b)$ are calculated by the Gauss-Chebyshev formula. For the first boundary value problem, $(7,12,13)$ can be discretized as

$$
\begin{aligned}
& \sum_{j=1}^{N} M_{i k}\left(y_{j}, y_{l}, p\right) \bar{u}_{i}\left(y_{j}, p\right)+\sum_{j=1}^{M} C_{i k}\left(\eta_{j}, y_{l}, p\right) \bar{F}_{i}\left(\eta_{j}, p\right) \\
& =\sum_{j=1}^{N} \bar{t}_{i}\left(y_{j}, p\right) \bar{U}_{i k}\left(y_{j}-y_{l}, p\right) h_{j} \\
& \sum_{j=1}^{N} K_{i k}\left(y_{j}, \tau_{m}, p\right) h_{j} \bar{u}_{i}\left(y_{j}, p\right)+\frac{b-a}{2 M} \\
& \quad \times \sum_{j=1}^{M}\left[\frac{A}{\eta_{j}-\tau_{m}}+\tau \overline{\bar{H}}_{i k}\left(\eta_{j}, \tau_{m}, p\right)\right] \bar{F}_{k}\left(\eta_{j}, p\right) \\
& =\bar{q}_{k}\left(\tau_{m}, p\right)-\sum_{j=1}^{N} \bar{t}_{i}\left(y_{j}, p\right) \bar{T}_{k i}^{+}\left(y_{j}, \tau_{m}, p\right) h_{j}, \\
& \frac{b-a}{2 M} \sum_{j=1}^{M} \bar{F}_{k}\left(\eta_{j}, p\right)=0
\end{aligned}
$$

where $k=1,2, l=1,2, \ldots, N, m=1,2, \ldots, M-1 ; h_{j}$ is the length of the element; $N$ is the number of boundary elements along $S ; \eta_{j}$ and $\tau_{m}$ are the zero points of first and second Chebyshev polynomials in $(a, b)$ and $M$ is the number of the zero point.

$$
\eta_{j}=\frac{b-a}{2} \cos \frac{\pi(2 j-1)}{2 M}+\frac{b+a}{2}, \quad \tau_{m}=\frac{b-a}{2} \cos \frac{\pi m}{M}+\frac{b+a}{2}
$$


and

$$
\begin{aligned}
& \bar{F}_{i}(x, p)=\frac{2}{b-a} \sqrt{(x-a)(b-x)} \Delta \bar{u}_{i, 1}(x, p), \\
& C_{i k}\left(\eta_{j}, y_{l}, p\right)=\frac{\pi(b-a)}{2 M} \int_{\eta_{j}}^{b} \bar{T}_{i k}^{+}\left(\eta^{*}, y_{l}\right) \mathrm{d} \eta^{*}, \\
& M_{i k}\left(y_{j}, y_{l}, p\right)=\left\{\begin{array}{l}
\bar{T}_{i k}\left(y_{j}, y_{l}, p\right) h_{j}, \quad j \neq l, \\
-\sum_{j=1, j \neq 1}^{N} \bar{T}_{i k}\left(y_{j}, y_{l}, p\right) h_{j}, \quad j=l .
\end{array}\right.
\end{aligned}
$$

The stress intensity factors in Laplace transform domain are determined by

$$
\begin{aligned}
& \bar{K}_{\mathrm{I}}(a, p)=\lim _{x \rightarrow a} \sqrt{2 \pi(x-a)} A \Delta \bar{u}_{2,1}(x, p), \\
& \bar{K}_{\mathrm{I}}(b, p)=-\lim _{x \rightarrow b} \sqrt{2 \pi(b-x)} A \Delta \bar{u}_{2,1}(x, p), \\
& \bar{K}_{\mathrm{II}}(a, p)=\lim _{x \rightarrow a} \sqrt{2 \pi(x-a)} A \Delta \bar{u}_{1,1}(x, p), \\
& \bar{K}_{\mathrm{II}}(b, p)=-\lim _{x \rightarrow b} \sqrt{2 \pi(b-x)} A \Delta \bar{u}_{1,1}(x, p) .
\end{aligned}
$$

By use of Equation (40), Formulae (41) can be rewritten as

$$
\begin{aligned}
& \bar{K}_{\mathrm{I}}(a, p)=A \sqrt{\frac{\pi(b-a)}{2}} \bar{F}_{2}(a, p), \\
& \bar{K}_{\mathrm{I}}(b, p)=-A \sqrt{\frac{\pi(b-a)}{2}} \bar{F}_{2}(b, p), \\
& \bar{K}_{\mathrm{II}}(a, p)=A \sqrt{\frac{\pi(b-a)}{2}} \bar{F}_{1}(a, p), \\
& \bar{K}_{\mathrm{II}}(b, p)=-A \sqrt{\frac{\pi(b-a)}{2}} \bar{F}_{1}(b, p),
\end{aligned}
$$

where $\bar{F}_{i}(a, p)$ and $\bar{F}_{i}(b, p)$ are computed by following interpolation formulae

$$
\begin{aligned}
& \bar{F}_{i}(a, p)=\frac{(-1)^{M}}{M} \sum_{j=1}^{M}(-1)^{j} \operatorname{tg} \frac{(2 j-1) \pi}{4 M} \cdot \bar{F}_{i}\left(\eta_{j}, p\right), \\
& \bar{F}_{i}(b, p)=\frac{1}{M} \sum_{j=1}^{M}(-1)^{j+1} \operatorname{ctg} \frac{(2 j-1) \pi}{4 M} \cdot \bar{F}_{i}\left(\eta_{j}, p\right) .
\end{aligned}
$$

In order to obtain the solution as a function of time we have to take the inversion of Laplace transform, but this can be done only numerically. In the present paper we choose the method of Miller and Guy suggested by [1,7]. The details can be seen in [7]. 


\section{Several numerical examples}

To show reliability of the method in this paper, some typical examples are calculated and their dynamic stress intensity factors are obtained in this section. The following numerical values are assigned to the constants describing the medium

$$
\nu=0.29, \quad \rho=7800 \mathrm{Kg} / \mathrm{m}^{3}, \quad \mu=8 \times 10^{10} \mathrm{~Pa} .
$$

EXAMPLE 1. Infinite plane with single crack subjected to impact loading

In this example, the dynamic stress intensity factors are computed for two cases; the results are consistent with those from [1].

EXAMPLE 2. Square with a central crack subjected to impact loading

EXAMPLE 3. Circular ring with a crack subjected to impact internal pressure

$$
R=2 r, \quad a=1.4 r, \quad b=1.6 r, \quad c=(b-a) / 2=0.1 r
$$

\section{Appendix}

The Green dynamic and static basic solutions are listed as follows respectively

$$
\begin{aligned}
& \bar{U}_{i k}(\eta-y)=\frac{1}{2 \pi \mu}\left(\bar{U}_{1} \delta_{i k}+\bar{U}_{2} r_{, i} r_{, k}\right), \\
& \bar{T}_{i k}(\eta, y)=\frac{1}{2 \pi r}\left\{\begin{array}{l}
{\left[\left(c_{1}^{2} / c_{2}^{2}-2\right) \bar{U}_{4}+2 \bar{U}_{2}\right] n_{i}(\eta) r_{, k}+\left(2 \bar{U}_{2}+\bar{U}_{3}\right) n_{k}(\eta) r_{, i}} \\
+\left[\left(2 \bar{U}_{2}+\bar{U}_{3}\right) \delta_{i k}+2\left(\bar{U}_{4}-\bar{U}_{3}-4 \bar{U}_{2}\right) r_{, i} r_{, k}\right] n_{j}(\eta) r_{, j}
\end{array}\right\}, \\
& U_{i k}(\eta-y)=\frac{1}{2 \pi \mu}\left(U_{1} \delta_{i k}+U_{2} r_{, i} r_{, k}\right), \\
& T_{i k}(\eta, y)=\frac{1}{2 \pi r}\left\{\begin{array}{l}
{\left[\left(c_{1}^{2} / c_{2}^{2}-2\right) U_{4}+2 U_{2}\right] n_{i}(\eta) r_{, k}+\left(2 U_{2}+U_{3}\right) n_{k}(\eta) r_{, i}} \\
+\left[\left(2 U_{2}+U_{3}\right) \delta_{i k}+2\left(U_{4}-U_{3}-4 U_{2}\right) r_{, i} r_{, k}\right] n_{j}(\eta) r_{, j}
\end{array}\right\},
\end{aligned}
$$

where

$$
\begin{aligned}
& \bar{U}_{1}=K_{0}\left(\frac{p r}{c_{2}}\right)+\frac{c_{2}}{p r} K_{1}\left(\frac{p r}{c_{2}}\right)-\left(\frac{c_{2}}{c_{1}}\right)^{2} \frac{c_{1}}{p r} K_{1}\left(\frac{p r}{c_{1}}\right), \\
& \bar{U}_{2}=\left(\frac{c_{2}}{c_{1}}\right)^{2}\left[K_{0}\left(\frac{p r}{c_{1}}\right)+2 \frac{c_{1}}{p r} K_{1}\left(\frac{p r}{c_{1}}\right)\right]-\left[K_{0}\left(\frac{p r}{c_{2}}\right)+2 \frac{c_{2}}{p r} K_{1}\left(\frac{p r}{c_{2}}\right)\right], \\
& \bar{U}_{3}=-\frac{p r}{c_{2}} K_{1}\left(\frac{p r}{c_{2}}\right), \quad \bar{U}_{4}=-\left(\frac{c_{2}}{c_{1}}\right)^{2} \frac{p r}{c_{1}} K_{1}\left(\frac{p r}{c_{1}}\right), \\
& U_{1}=-\frac{1}{2}\left(\frac{1+c_{2}^{2}}{c_{1}^{2}}\right) \ln r, \quad U_{2}=\frac{1}{2}\left(\frac{1-c_{2}^{2}}{c_{1}^{2}}\right), \\
& U_{3}=-1, \quad U_{4}=-\frac{c_{2}^{2}}{c_{1}^{2}},
\end{aligned}
$$




$$
r_{k}=\eta_{k}-y_{k}, \quad r=\sqrt{r_{k} r_{k}}, \quad r_{, k}=r_{k} / r, \quad k=1,2 .
$$

\section{References}

1. G.C. Sih (ed), Mechanics of Fracture, Vol 4, Noordoof International Publishing, Leyden (1977).

2. G.C. Sih and E.P. Chen, Normal and shear impact of layered composite with a crack: dynamic stress intensification, J. Appl. Mech. 47 (1980) 351-358.

3. H. Nozaki, Y. Shindo and A. Atsumi, Impact response of a cylinder composite with a penny-shaped crack, Int. J. Solids Structures 22, No. 10 (1986) 1137-1147.

4. S. Itou, Dynamics stress intensity factors around a rectangular crack in an infinite plate under impact load, Eng. Fracture Mech. 16, No. 2 (1987) 247-256.

5. W.H. Tai and KR. Li, Elastodynamic repose of a finite strip with two coplanar cracks under impact load, Eng. Fracture Mech. 27, No. 4 (1987) 379-390.

6. M.F. Kanninen, A critical appraisal of solution techniques in dynamic fracture mechanics, Number. Math. Fracture Mech. (Ed, by A.R. Luxmore and D.R. J. Owen), (1978) 612-634, Swansea.

7. T.Y. Fan and H.G. Hahn, An application of the boundary integral equation method to dynamic fracture mechanics, Eng. Fracture Mech. 21, No. 2 (1985) 307-313.

8. J. Sladek and V. Sladek, A boundary integral equation method for dynamic crack problems, Eng. Fracture Mech. 27, No. 3 (1987) 269-277.

9. F. Chirino and J. Dominguez, Dynamic analysis of cracks using boundary element method, Eng. Fracture Mech., 34, No. 5/6 (1989) 1051-1061.

10. T.A. Cruse, Fracture mechanics, Boundary Element Methods in Mechanics, D.E. Beskos, ed., North Holland, (1987) 333-365.

11. V. Sladek and J. Sladek, Transient elastodynamic three-dimensional problems in cracked bodies, Appl. Math. Modeling, 8, No 2 (1984) 2-10.

12. CH. Zhang and J.D. Achenbach, Scattering by multiple crack configurations, ASME, J. Appl. Mech., 55, No 1 (1988) 104-110.

13. $\mathrm{CH}$. Zhang and J.D. Achenbach, A new boundary integral equation methods formulation for elastodynamic and elastostatic crack analysis, ASME, J. Appl. Mech. 56 (1989) 284-290.

14. CH. Zhang, A novel derivation of non-hypersingular time-domain BIEs for transient elastodynamic crack analysis. Int. J. Solids Struct. 28 (1991) 267-281.

15. CH. Zhang and D. Gross, 3-D elastodynamic crack analysis by non-hypersingular BIEM, Comput. Mech. 9 (1992) 137-152.

16. $\mathrm{CH}$. Zhang and D. Gross, A non-hypersingular time-domain BIEM for 3-D transient elastodynamic crack analysis 36 (1993) 2997-3017.

17. F. Erdogan, Mixed boundary value problem, Mechanics Today, S. Nemat-Nasser, ed. 4, (1978).

18. G.N. Watson, Theory of Bessel Functions, Cambridge University Press, 2nd edition (1952). 